\title{
SAZONALIDADE, COMPOSIÇÃO E APORTE DE NUTRIENTES DA SERAPILHEIRA EM FRAGMENTO DE MATA ATLÂNTICA ${ }^{1}$
}

\author{
Silvana Andreoli Espig 2 , Fernando José Freire ${ }^{3}$, Luiz Carlos Marangon ${ }^{4}$, Rinaldo Luiz Caraciolo \\ Ferreira $^{4}$, Maria Betânia Galvâo dos Santos Freire ${ }^{3}$ e Darci Bacelar Espig ${ }^{3}$
}

\begin{abstract}
RESUMO - Este trabalho objetivou quantificar a deposição de serapilheira, avaliar a influência da precipitação nessa deposição, determinar os teores e calcular o aporte de nutrientes em função do tempo e dos componentes vegetais. A pesquisa foi conduzida em um remanescente de Mata Atlântica na região metropolitana de Recife, PE. A deposição de serapilheira foi de 10,07 t/ha/ano, e o componente folha contribuiu com 6,74 t/ha/ano (66,9\% do total). A deposição de serapilheira foi maior nos períodos secos, e a sazonalidade influenciou o componente folha da serapilheira. Os teores de $\mathrm{K}$ foram menores nos períodos chuvosos, nos resíduos folhas e miscelânea. Os teores dos nutrientes Ca, K, Mg e P na serapilheira foram de 15,73; 4,74; 2,42; e 0,50 g/ $\mathrm{kg}$, respectivamente. $\mathrm{O}$ aporte de nutrientes via serapilheira foi de 170,$7 ; 26,4 ; 5 ; \mathrm{e} 49,7 \mathrm{~kg} / \mathrm{ha} / \mathrm{ano}$ para $\mathrm{Ca}, \mathrm{Mg}, \mathrm{P}$ e K, respectivamente.
\end{abstract}

Palavras-chave: Floresta Atlântica, serapilheira e distribuição temporal.

\section{LITTER SEASONALITY, COMPOSITION AND NUTRIENT INPUT IN REMNANT OF ATLANTIC FOREST IN THE STATE OF PERNAMBUCO, $B R A Z I L$}

\begin{abstract}
This research aimed to evaluate the amount of litter fall, the seasonal distribution and the influence of precipitation on the fall. It also intended to determine the nutrient contents and estimated the nutrient input according to time and plant structure. The research was carried out in an Atlantic Forest remnant in Recife-PE metropolitan region. The litter fall was equal to 10.07 tons/ha/year, and the leaf component contributed with 6.74 tons/ha/year (66.9\% of the total). Litter fall was higher during the dry season. Seasonality influenced the leaf component of the litter fall. The amount of $K$ was low in the rainy season for the leaf and miscellaneous components. The contents of nutrients in $\mathrm{g} / \mathrm{kg}$ in the litter were $\mathrm{Ca}, 15.73 ; \mathrm{K}, 4.74 ; \mathrm{Mg}$, 2.42; and $P, 0.50$. The input of nutrients through the litter was $170.7 ; 26.4 ; 5.2$; and $49.7 \mathrm{~kg} / \mathrm{ha} / \mathrm{year}$ for $\mathrm{Ca}, \mathrm{Mg}, \mathrm{P}$ and $\mathrm{K}$, respectively.
\end{abstract}

Keywords: Atlantic forest, litter and temporal distribution.

\section{INTRODUÇÃO}

Os processos de absorção de nutrientes pelas plantas, translocação interna entre os tecidos vegetais e transferência dos nutrientes acumulados na serapilheira para o solo, a atmosfera e a hidrosfera representam a ciclagem de nutrientes. Essa ciclagem é um processo essencial na sustentabilidade dos sistemas e para a manutenção da vida no planeta (ANDRADE et al., 1999).
Estudos de ciclagem de nutrientes em florestas naturais são muito complexos, por envolver ciclos, como o geoquímico e o biológico (ciclo bioquímico e biogeoquímico) (PRITCHETT, 1979). Para tanto, estuda-se, normalmente, esse complexo por partes, direcionando-o à porção que mais contribui para a absorção e transferência de nutrientes, que é a serapilheira (ciclo biogeoquímico) (CUNHA et al., 1993; SCHUMACHER et al., 2003; FERREIRA et al., 2007).

\footnotetext{
${ }^{1}$ Recebido em 11-12-2007 e aceito para publicação em 23.06.2009.

${ }^{2}$ Instituto de Pesquisas Espaciais (INPE), São José dos Campos, SP. E-mail: <sil.andreoli@ gmail.com>.

${ }_{3}^{3}$ Departamneto de Agronomia da Universidade Federal Rural de Pernambuco (UFRPE), Recife, PE. E-mail: <f.freire@ depa.ufrpe.br>, <betania@depa.ufrpe.br>e <despig@uol.com.br>.

${ }^{4}$ Departamento de Ciências Florestais da Universidade Federal Rural de Pernambuco(UFRPE), Recife, PE. E-mail: <rinaldo@ dcfl.ufrpe.br> e<marangon@dcfl.ufrpe.br>.
} 
A serapilheira é formada por material vegetal da parte aérea das plantas, como folhas, frutos, sementes, flores, galhos e cascas, em que as folhas, geralmente, representam a maior fração. Existem vários fatores que interferem na deposição da serapilheira em ecossistemas florestais, e os que mais se destacam são o clima, o solo, as características genéticas das plantas, a idade do povoamento e a densidade de plantas (GONZALEZ e GALLARDO, 1982). Das variáveis climáticas, temperatura e precipitação são as que mais afetam a deposição de serapilheira em ecossistemas florestais. A maior produção pode estar relacionada com períodos secos (GOLLEY et al., 1978; NUNES, 1980) ou com períodos úmidos (CUNHA et al., 1993; DIAS e OLIVEIRA FILHO, 1997; HINKEL e PANITZ, 1999). A temperatura correlaciona-se positivamente com a formação de serapilheira, ou seja, florestas de regiões temperadas acumulam menos serapilheira do que as de regiões tropicais (HAAG, 1985).

Trabalhos reunidos por Haag (1985) mostraram que a produção de serapilheira em florestas tropicais situa-se na faixa de 7 a 12 t/ha/ano de matéria seca, e o componente "folha" representa a maior proporção em relação à soma dos componentes "ramos" e "outros materiais". Freitas (1990), estudando 11 matas úmidas costeiras em Pernambuco, encontrou deposição entre 8 e 28 t/ha/ano, observou alta variação espacial entre matas. Nunes (1980), pesquisando várias formações florestais em regiões tropicais, observou que a deposição de serapilheira variou entre 4,0 e 25,3 t/ ha/ano.

A manutenção da exuberância das florestas tropicais úmidas ocorre em função da sincronia entre disponibilidade e demanda de nutrientes das espécies, tendo a diversidade biológica e a deposição da serapilheira como elementos essenciais para o equilíbrio e sustentabilidade dos ecossistemas florestais. Considerando que é a serapilheira a porção que mais contribui para a transferência de nutrientes em ecossistemas florestais, muitos trabalhos foram realizados sobre teores de elementos nutricionais nesse componente do ciclo biogeoquímico (GOLLEY et al., 1978; NUNES, 1980; HAAG, 1985).

Este trabalho objetivou quantificar a deposição de serapilheira, avaliar a sazonalidade e influência da precipitação nessa deposição e determinar os teores e o aporte de nutrientes em um fragmento de Mata Atlântica em Pernambuco, cuja vegetação é dominada por diferentes espécies características da região e influenciadoras dos atributos químicos e físicos dos latossolos predominantes nas zonas úmidas costeiras do Nordeste.

\section{MATERIAL E MÉTODOS}

A área em estudo localiza-se na região metropolitana de Recife, PE, e constitui remanescente de Mata Atlântica, preservado por se encontrar em área militar, enquadrando-se como Floresta Ombrófila Densa de Encosta (VELOSO e GÓES FILHO, 1982). O solo no fragmento foi classificado como Latossolo Amarelo Distrófico (EMBRAPA, 1999).

Coletaram-se os componentes da serapilheira, como folhas, frutos, flores, galhos (menores que $1 \mathrm{~cm}$ de diâmetro) e cascas, por meio de 40 caixas coletoras, instaladas no centro das parcelas de $10 \times 25 \mathrm{~m}$, utilizadas para estudo fitossociológico. Essas caixas foram confeccionadas em isopor de alta densidade nas dimensões de $1 \mathrm{~m} \mathrm{x}$ $1 \mathrm{~m}$ e altura de $0,15 \mathrm{~m}$, tendo na base uma tela de náilon de malha de $2 \mathrm{~mm}$. As caixas foram suspensas a aproximadamente $1 \mathrm{~m}$ de altura do solo.

A coleta da serapilheira foi feita mensalmente, de janeiro a dezembro de 2002. Colocou-se o material em sacos de papel, a qual foi levado à estufa de circulação forçada de ar a $65^{\circ} \mathrm{C}$ até massa constante. Em seguida, o material foi retirado da estufa e triado, separando-se as folhas do restante (flores, frutos, galhos e cascas), pesado e moído.

Os nutrientes $\mathrm{Ca}, \mathrm{Mg}, \mathrm{P}$ e $\mathrm{K}$ foram extraídos por digestão nítrico-perclórica (BATAGLIA et al., 1983). Posteriormente, o Ca e o Mg foram determinados por espectrofotometria de absorção atômica, o P foi dosado por colorimetria (BRAGA e DEFELIPO, 1974) e o K, determinado por fotometria de chama.

As deposições mensais da serapilheira e os teores dos nutrientes foram correlacionados com as precipitações pluviais médias mensais. Os dados de deposição de serapilheira, teores nutricionais e aporte de nutrientes foram tratados estatisticamente em blocos casualizados, utilizando-se análise de variância e comparação de médias pelo teste de Scott-Knott para o fator mês e o teste $\mathrm{F}$ para os componentes da serapilheira (folha e miscelânea). 


\section{RESULTADOS E DISCUSSÃO}

O fragmento apresentou valores da fitomassa seca de folha na serapilheira de $6,74 \mathrm{t} / \mathrm{ha} /$ ano e da miscelânea de 3,33 t/ha/ano, totalizando um aporte anual de 10,07 t/ha/ano (Tabela 1). Esse resultado foi próximo dos encontrados por Golley et al. (1978) e Nunes (1980), respectivamente de 11,35 e 11,20 t/ha/ano. O componente folha da serapilheira representou $66,9 \%$ da fitomassa total. No entanto, a deposição de serapilheira mostrou-se variável ao longo do ano, e a fitomassa da componente folha foi cerca de duas vezes a da componente miscelânea (Tabela 1).

A quantidade de serapilheira depositada foi inversamente relacionada $(\mathrm{r}=-0,549 \mathrm{p}<5 \%)$ com a precipitação média anual representativa da condição climática local (Figura 1A). Esse resultado tem sido observado em alguns trabalhos realizados em florestas tropicais (GOLLEY et al., 1978; NUNES, 1980). Contudo, outros autores (CUNHA et al., 1993; DIAS e OLIVEIRA FILHO, 1997; HINKEL e PANITZ, 1999) encontraram que, em períodos úmidos, a deposição de serapilheira foi maior. Porém, nesses estudos, outras variáveis influenciaram tal comportamento, a exemplo da temperatura.

Quando foram analisados os componentes da serapilheira separadamente (folha e miscelânea), observou-se o mesmo comportamento para a fitomassa do componente folha, possivelmente porque esta compõe a maior proporção da fitomassa total da serapilheira. Entretanto, não houve correlação significativa entre o comportamento da fitomassa da miscelânea e a precipitação (Figura 1B).

A análise da variância da deposição de serapilheira mostrou influência altamente significativa $(\mathrm{p}<0,01)$ dos fatores mês e componente e da interação (Tabela 1). A deposição depende do mês só para o componente folha. Considerando que o mês reflete a precipitação, então os resultados da análise da variância são coerentes com o das correlações (Figura 1AB). No entanto, o componente folha só foi igual estatisticamente ao componente miscelânea no mês de janeiro, por causa da acentuada diminuição da fitomassa de folha (417 $\mathrm{kg} / \mathrm{ha}$ ) no período chuvoso (Tabela 1 ).

Correlações realizadas entre os teores dos nutrientes nos componentes folha e miscelânea da serapilheira e a precipitação mostraram que os teores de $\mathrm{Ca}, \mathrm{Mg}$
Tabela 1 - Fitomassa mensal dos componentes folha e miscelânea da serapilheira, análise da variância e coeficiente de variação em fragmento de Mata Atlântica, em Pernambuco.

Table 1 - Leaf and miscellaneous components of litter fall in a year in an Atlantic Forest fragment in Pernambuco, Brazil.

\begin{tabular}{|c|c|c|}
\hline \multirow[t]{2}{*}{ Fator } & \multicolumn{2}{|c|}{ Componente } \\
\hline & Folha & Miscelânea \\
\hline & \multicolumn{2}{|c|}{$\mathrm{kg} / \mathrm{ha}$} \\
\hline \multicolumn{3}{|l|}{ Mês } \\
\hline Janeiro & $417 \mathrm{Ac}$ & $295 \mathrm{Aa}$ \\
\hline Fevereiro & $658 \mathrm{Ab}$ & $365 \mathrm{Ba}$ \\
\hline Março & $455 \mathrm{Ac}$ & $275 \mathrm{Ba}$ \\
\hline Abril & $466 \mathrm{Ac}$ & $218 \mathrm{Ba}$ \\
\hline Maio & $426 \mathrm{Ac}$ & $247 \mathrm{Ba}$ \\
\hline Junho & $534 \mathrm{Ab}$ & $292 \mathrm{Ba}$ \\
\hline Agosto & $588 \mathrm{Ab}$ & $299 \mathrm{Ba}$ \\
\hline Setembro & $845 \mathrm{Aa}$ & $208 \mathrm{Ba}$ \\
\hline Outubro & $950 \mathrm{Aa}$ & $379 \mathrm{Ba}$ \\
\hline Novembro & $796 \mathrm{Aa}$ & $418 \mathrm{Ba}$ \\
\hline Dezembro & $601 \mathrm{Ab}$ & $338 \mathrm{Ba}$ \\
\hline \multirow[t]{2}{*}{ Total (kg/ha/ano) } & 6.736 & 3.334 \\
\hline & & \\
\hline Mês & \multicolumn{2}{|c|}{$9,30^{* * *}$} \\
\hline Componente & \multicolumn{2}{|c|}{$200,47^{* * *}$} \\
\hline Mês * Componente & \multicolumn{2}{|c|}{$4,96^{* * *}$} \\
\hline C.V. $(\%)$ & \multicolumn{2}{|c|}{70,76} \\
\hline
\end{tabular}

e P não são influenciados pela precipitação (Figura 2). No entanto, o teor de $\mathrm{K}$ foi altamente influenciado, apresentando correlações significativa e negativa com a precipitação $(r=-0,781 \mathrm{p}<0,1 \%)$, ou seja, os teores de K na folha são maiores quando a precipitação é baixa e vice-versa.

Segundo Mengel e Kirkby (1982), o K é um dos íons mais facilmente lixiviados das copas das árvores, por não ser componente estrutural de nenhum composto orgânico. O Ca, o Mg e o P, por fazerem parte de compostos orgânicos, não foram influenciados pela precipitação, ou seja, não foram lixiviados diretamente da superfície dos componentes, como o K. Os resultados do K, por sua vez, podem indicar que esse elemento é facilmente removido com as chuvas, indicando que isso ocorra também nos diversos componentes da parte aérea da vegetação dos ecossistemas florestais.

Os teores médios de Ca e $\mathrm{Mg}$ na serapilheira desse fragmento foram de 15,73 e 2,42 $\mathrm{g} / \mathrm{kg}$, respectivamente (Tabela 2), e corroboraram os encontrados de Golley

R. Árvore, Viçosa-MG, v.33, n.5, p.949-956, 2009 

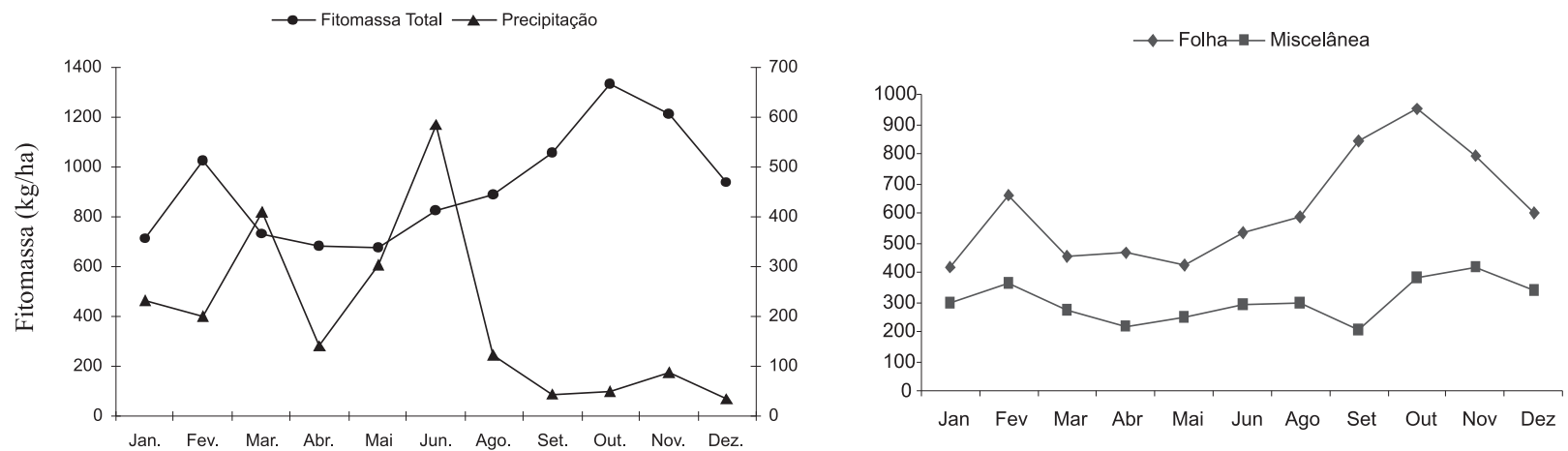

Figura 1 - Fitomassa total (A) e fitomassa das folhas e da miscelânea (B) em função do tempo e da precipitação em fragmento de Mata Atlântica, em Pernambuco.

Figure 1 - Litter fall (A) and leaf and miscellaneous components of litter $(B)$ during a year in a remaining Atlantic Forest in Pernambuco, Brazil.

Tabela 2 - Teores médios mensais de cálcio, magnésio, fósforo e potássio, análise da variância e coeficiente de variação nos componentes da serapilheira (folha e miscelânea) em fragmento de Mata Atlântica, em Pernambuco.

Table 2 - Calcium, magnesium, phosphorus and potassium contents in leaf and miscellaneous components of litter in a remaining Atlantic Forest in Pernambuco, Brazil.

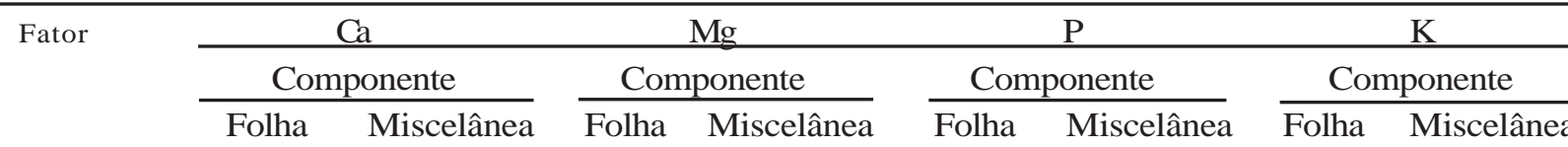

Mês

$\begin{array}{lllll}\text { Janeiro } & 16,34 \mathrm{Aa} & 15,61 \mathrm{Ac} & 2,41 \mathrm{a} & 2,33 \mathrm{a} \\ \text { Fevereiro } & 16,45 \mathrm{Aa} & 13,68 \mathrm{Bd} & 2,52 \mathrm{a} & 2,35 \mathrm{a} \\ \text { Março } & 16,63 \mathrm{Aa} & 15,14 \mathrm{Bc} & 2,43 \mathrm{a} & 2,32 \mathrm{a} \\ \text { Abril } & 15,09 \mathrm{Ab} & 14,54 \mathrm{Ac} & 2,46 \mathrm{a} & 2,33 \mathrm{a} \\ \text { Maio } & 15,97 \mathrm{Aa} & 14,82 \mathrm{Ac} & 2,38 \mathrm{~b} & 2,25 \mathrm{a} \\ \text { Junho } & 17,12 \mathrm{Aa} & 16,33 \mathrm{Ab} & 2,54 \mathrm{a} & 2,33 \mathrm{a} \\ \text { Agosto } & 16,69 \mathrm{Ba} & 18,55 \mathrm{Aa} & 2,46 \mathrm{a} & 2,42 \mathrm{a} \\ \text { Setembro } & 15,77 \mathrm{Ab} & 14,65 \mathrm{Ac} & 2,35 \mathrm{~b} & 2,26 \mathrm{a} \\ \text { Outubro } & 16,93 \mathrm{Aa} & 16,21 \mathrm{Ab} & 2,52 \mathrm{a} & 2,49 \mathrm{a} \\ \text { Novembro } & 14,67 \mathrm{Ab} & 13,57 \mathrm{Ad} & 2,25 \mathrm{~b} & 2,18 \mathrm{a} \\ \text { Dezembro } & 16,25 \mathrm{Aa} & 15,06 \mathrm{Ac} & 2,30 \mathrm{~b} & 2,14 \mathrm{a}\end{array}$

$\begin{array}{ll}0,34 \mathrm{Ba} & 0,68 \mathrm{Aa} \\ 0,38 \mathrm{Ba} & 0,72 \mathrm{Aa} \\ 0,35 \mathrm{Ba} & 0,66 \mathrm{Aa} \\ 0,39 \mathrm{Ba} & 0,67 \mathrm{Aa} \\ 0,34 \mathrm{Ba} & 0,54 \mathrm{Ab} \\ 0,42 \mathrm{Aa} & 0,50 \mathrm{Ab} \\ 0,40 \mathrm{Aa} & 0,49 \mathrm{Ab} \\ 0,37 \mathrm{Ba} & 0,56 \mathrm{Ab} \\ 0,38 \mathrm{Ba} & 0,67 \mathrm{Aa} \\ 0,40 \mathrm{Ba} & 0,70 \mathrm{Aa} \\ 0,37 \mathrm{Ba} & 0,76 \mathrm{Aa}\end{array}$

$3,78 \mathrm{Bb}$

$3,28 \mathrm{Bb}$

$3,42 \mathrm{Bb}$

$3,81 \mathrm{Bb}$

$3,19 \mathrm{Ab}$

2,64 Ab

3,98 Ab

$5,20 \mathrm{Ba}$

$5,91 \mathrm{Aa}$

$3,88 \mathrm{Ab}$

6,13 Aa

5,59 Ac

4,64 Ac

5,32 Ac

$4,94 \mathrm{Ac}$

$3,89 \mathrm{Ad}$

2,77 Ae

$3,80 \mathrm{Ad}$

6,45 Ab

$8,10 \mathrm{Aa}$

5,74 Ac

7,97 Aa

F

\begin{tabular}{|c|c|c|c|c|}
\hline Mês & $9,01^{* * * *}$ & $3,28^{* * *}$ & $3,22^{* * * *}$ & $35,94^{* * * *}$ \\
\hline Componente & $20,11^{* * * *}$ & $53,87^{* * * *}$ & $295,02^{* * *}$ & $85,16^{* * *}$ \\
\hline Mês * Comp. & $2,80^{* *}$ & $1,13^{\mathrm{ns}}$ & $4,20^{* * * *}$ & $2,85^{* *}$ \\
\hline C.V. (\%) & 18,58 & 19,12 & 44,02 & 43,10 \\
\hline
\end{tabular}

Médias seguidas da mesma letra minúscula na coluna não diferem entre si, pelo teste de Scott-Knott a 5\%. Médias seguidas da mesma letra maiúscula na linha não diferem entre si, pelo teste de $\mathrm{F}$ a $5 \%$.

*,** e $* * *$ significativos a $5 ; 1 ;$ e $0,1 \%$, respectivamente, pelo teste de $\mathrm{F}$. ${ }^{\mathrm{ns}}$ não-significativo.

R. Árvore,Viçosa-MG, v.33, n.5, p.949-956, 2009 
et al. (1978), que foram de $14,17 \mathrm{~g} / \mathrm{kg}$ para Ca e 2,04 g/kg para Mg. Entretanto, Sampaio et al. (1988) encontraram teores menores em floresta tropical úmida, tanto para $\mathrm{Ca}(5,4 \mathrm{~g} / \mathrm{kg})$ quanto para $\mathrm{Mg}(1,93 \mathrm{~g} / \mathrm{kg})$. Todavia, os teores de $\mathrm{P}$ e $\mathrm{K}$ deste trabalho $(0,50 \mathrm{e}$ $4,74 \mathrm{~g} / \mathrm{kg}$, respectivamente) (Tabela 2) assemelharam-se aos de Sampaio et al. (1988), que foram de 0,70 g/kg para $\mathrm{P} \mathrm{e} \mathrm{6,1} \mathrm{g/kg} \mathrm{para} \mathrm{K} \mathrm{do} \mathrm{que} \mathrm{os} \mathrm{de} \mathrm{Golley} \mathrm{et} \mathrm{al.} \mathrm{(1978),}$ que foram de $1,03 \mathrm{~g} / \mathrm{kg}$ para $\mathrm{P}$ e $7,33 \mathrm{~g} / \mathrm{kg}$ para $\mathrm{K}$.

$\mathrm{A}$ análise da variância dos teores de $\mathrm{Ca}, \mathrm{Mg}$, $\mathrm{P}$ e K na serapilheira mostrou que há diferença altamente significativa ( $\mathrm{p}<0,001)$ quanto aos fatores mês e componente. A interação desses fatores também foi significativa para $\mathrm{Ca}(\mathrm{p}<0,01), \mathrm{P}(\mathrm{p}<0,001)$ e K $(p<0,01)$, portanto os teores desses nutrientes nos componentes folha e miscelânea foram dependentes do mês de coleta da serapilheira. No entanto, com relação a $\mathrm{Mg}(\mathrm{p}<0,05)$ os teores na folha foram maiores do que na miscelânea, independentemente do mês (Tabela 2).
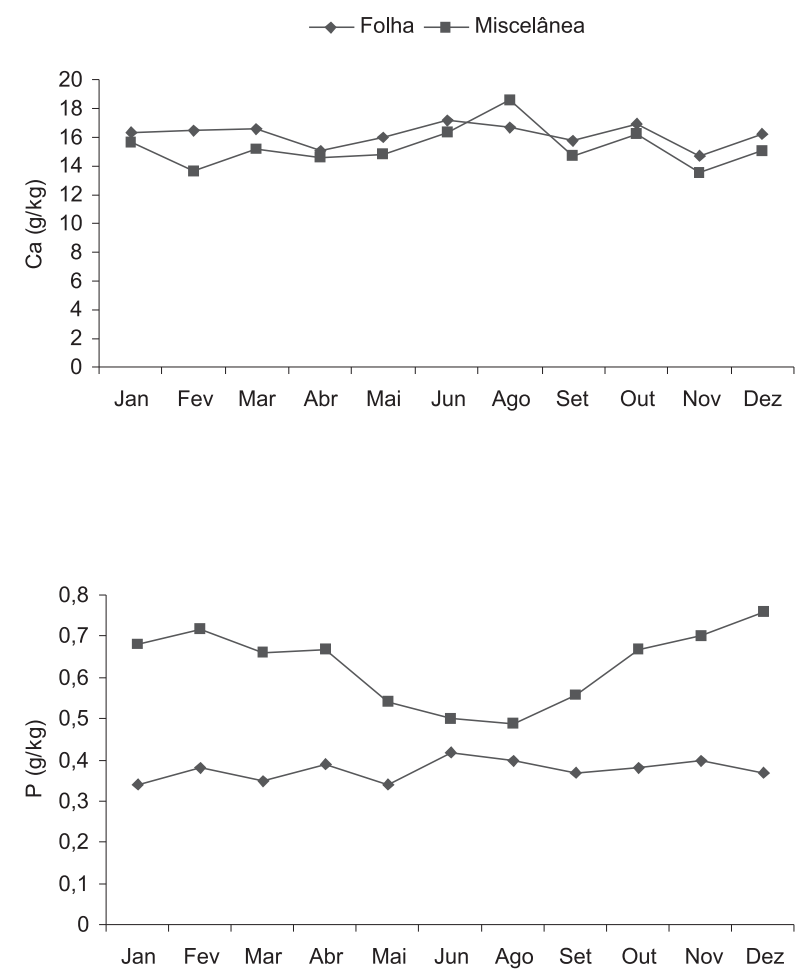

Os teores de Ca no componente folha só foram estatisticamente diferentes ( $p>0,05)$ dos da miscelânea nos meses de fevereiro, março e agosto (Tabela 2). Como os teores de Ca não se correlacionaram com a precipitação (Figura 2), é provável que o aporte de folhas de espécies com maiores teores desse nutriente tenha contribuído mais na composição de folhas da serapilheira em fevereiro e março e menos em agosto. Do mesmo modo, pode-se considerar que a qualidade da miscelânea também pode ter influenciado as diferenças nos teores $\mathrm{Ca}$ naqueles meses, com variados teores desse nutriente nos constituintes da miscelânea (cascas, flores, frutos, galhos).

Os teores de P e K são maiores no componente miscelânea do que folha (Tabela 2). Para P, só nos meses de junho e agosto esses teores são estatisticamente semelhantes $(\mathrm{p}>0,05)$, porque nesses meses a miscelânea apresentou os menores teores $(0,50$ e $0,49 \mathrm{~g} / \mathrm{kg}$, respectivamente) (Tabela 2). É provável que a qualidade da miscelânea tenha sido responsável por essa diminuição
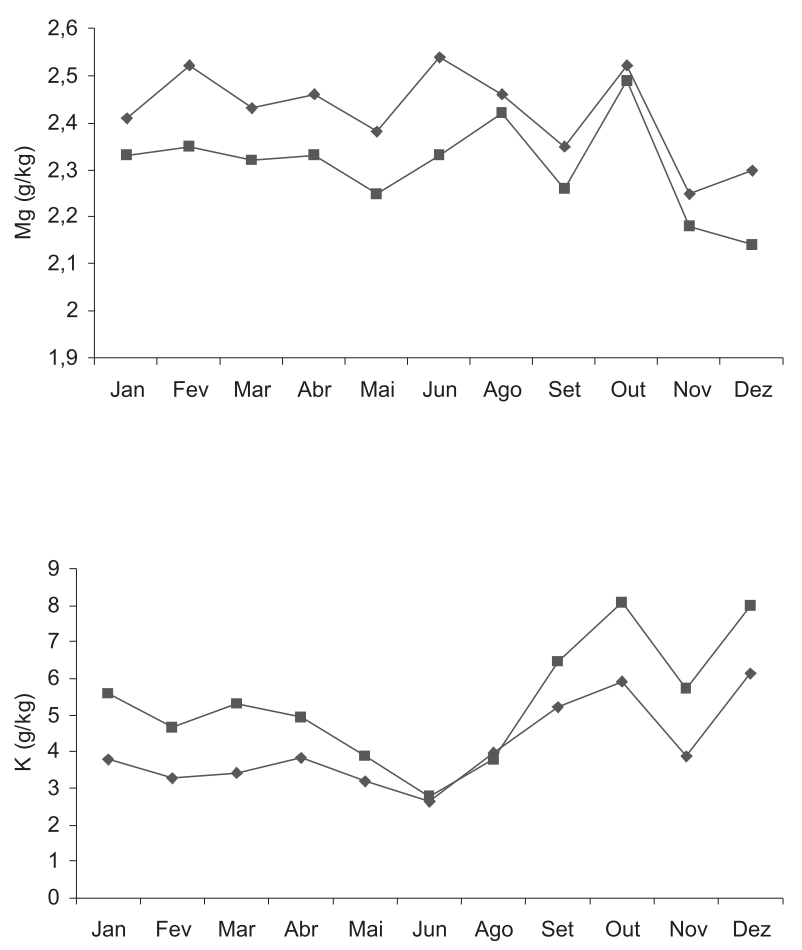

Figura 2 - Teores de cálcio, magnésio, fósforo e potássio nos componentes folha e miscelânea da serapilheira, em função do tempo e da precipitação em fragmento de Mata Atlântica, em Pernambuco.

Figure 2 - Calcium, magnesium, phosphorus and potassium contents in leaf and miscellaneous components in a remaining Atlantic Forest in Pernambuco, Brazil. 
dos teores de $\mathrm{P}$, com menores contribuições de flores, constituintes mais ricos em $\mathrm{P}$, aliada ao maior aporte desse nutriente na serapilheira por folhas de espécies com elevados teores de P.

A diminuição dos teores de $\mathrm{K}$ nos componentes folha e miscelânea nos meses mais chuvosos relaciona-se, provavelmente, com a lixiviação desse nutriente diretamente da superfície foliar, pela correlação negativa e significativa do teor de K com a precipitação (Figura 2). Isso indica que a qualidade do material que compõe a serapilheira e a incidência de chuvas podem influenciar os teores de $\mathrm{K}$ nos componentes estudados.

Os aportes médios mensais de $\mathrm{Ca}$ e $\mathrm{Mg}$ da componente folha da serapilheira são estatisticamente maiores $(\mathrm{p}<0,001)$ do que da componente miscelânea, independentemente do mês (Tabela 3), sugerindo que a ciclagem desses nutrientes ocorre basicamente pela componente folha da serapilheira. Variações no aporte de Ca e $\mathrm{Mg}$ através da folha em função do mês mostram que os maiores aportes ocorrem nos meses de setembro a novembro, provavelmente coincidindo com a época de maior deciduosidade de espécies que apresentam elevados teores de Ca e Mg. Entretanto, essa é a época em que a deposição de folhas da serapilheira é mais elevada, por ser o período mais seco do ano (Figura 1B). O aporte desses nutrientes foi através da miscelânea, independentemente do mês, porque a deposição desse componente não se correlacionou com a precipitação (Figura 1B).

Contrariamente ao que ocorre com $\mathrm{Ca}$ e $\mathrm{Mg}$, a magnitude do aporte de $\mathrm{P}$ da componente miscelânea é estatisticamente semelhante $(\mathrm{p}<0,05)$ à componente folha da serapilheira (Tabela 3 ). Isso sugere que o aporte de P na ciclagem de nutrientes nesse fragmento não depende, exclusivamente, da deposição de folhas, mas também de flores, frutos, casca e galhos (miscelânea). Como ocorreu com $\mathrm{Ca}$ e $\mathrm{Mg}$, os maiores aportes de $\mathrm{P}$, através da componente folha, foram nos meses mais secos, pela correlação negativa e significativa entre deposição de folhas e precipitação (Figura 1B). Na componente miscelânea, como a correlação com a precipitação é não significativa, os maiores aportes em determinados meses parecem mais relacionados com a qualidade do material vegetal depositado.

Tabela 3-Aportes médios mensais de cálcio, magnésio, fósforo e potássio, análise da variância e coeficiente de variação nos componentes da serapilheira (folha e miscelânea) em fragmento de Mata Atlântica, em Pernambuco.

Table 3 - Calcium, magnesium, phosphorus and potassium input in leaf and miscellaneous components in a remaining Atlantic Forest in Pernambuco, Brazil.

\begin{tabular}{|c|c|c|c|c|c|c|c|c|}
\hline \multirow[t]{3}{*}{ Fator } & \multicolumn{2}{|r|}{$\mathrm{Ca}$} & \multicolumn{2}{|r|}{$\mathrm{Mg}$} & \multicolumn{2}{|r|}{$\mathrm{P}$} & \multicolumn{2}{|r|}{$\mathrm{K}$} \\
\hline & \multicolumn{2}{|c|}{ Componente } & \multicolumn{2}{|c|}{ Componente } & \multicolumn{2}{|c|}{ Componente } & \multicolumn{2}{|c|}{ Componente } \\
\hline & Folha & Miscelânea & Folha & Miscelânea & Folha & Miscelânea & Folha & $\overline{\text { Miscelânea }}$ \\
\hline \multicolumn{9}{|l|}{ Mês } \\
\hline Janeiro & $6,74 \mathrm{Ad}$ & $4,42 \mathrm{Ba}$ & $1,00 \mathrm{Ad}$ & $0,65 \mathrm{Ba}$ & $0,13 \mathrm{Ab}$ & $0,19 \mathrm{Ab}$ & $1,49 \mathrm{Ac}$ & $1,67 \mathrm{Ab}$ \\
\hline Fevereiro & $11,06 \mathrm{Ab}$ & $4,84 \mathrm{Ba}$ & $1,80 \mathrm{Ab}$ & $0,87 \mathrm{Ba}$ & $0,27 \mathrm{Aa}$ & $0,30 \mathrm{Aa}$ & $2,25 \mathrm{Ac}$ & $1,96 \mathrm{Ab}$ \\
\hline Março & $7,77 \mathrm{Ad}$ & $3,84 \mathrm{Ba}$ & $1,20 \mathrm{Ad}$ & $0,60 \mathrm{Ba}$ & $0,16 \mathrm{Ab}$ & $0,20 \mathrm{Ab}$ & $1,50 \mathrm{Ac}$ & $1,57 \mathrm{Ab}$ \\
\hline Abril & 6,97 Ad & $3,14 \mathrm{Ba}$ & $1,21 \mathrm{Ad}$ & $0,49 \mathrm{Ba}$ & $0,18 \mathrm{Ab}$ & $0,15 \mathrm{Ab}$ & $1,81 \mathrm{Ac}$ & $1,02 \mathrm{Ab}$ \\
\hline Maio & $6,83 \mathrm{Ad}$ & $3,42 \mathrm{Ba}$ & $1,08 \mathrm{Ad}$ & $0,51 \mathrm{Ba}$ & $0,14 \mathrm{Ab}$ & $0,13 \mathrm{Ab}$ & $1,30 \mathrm{Ac}$ & $1,04 \mathrm{Ab}$ \\
\hline Junho & $9,10 \mathrm{Ac}$ & $4,63 \mathrm{Ba}$ & $1,48 \mathrm{Ac}$ & $0,66 \mathrm{Ba}$ & $0,22 \mathrm{Ab}$ & $0,15 \mathrm{Ab}$ & $1,44 \mathrm{Ac}$ & $0,80 \mathrm{Ab}$ \\
\hline Agosto & $9,76 \mathrm{Ac}$ & $4,91 \mathrm{Ba}$ & $1,45 \mathrm{Ac}$ & $0,69 \mathrm{Ba}$ & $0,23 \mathrm{Ab}$ & $0,15 \mathrm{Ab}$ & $2,41 \mathrm{Ac}$ & $1,38 \mathrm{Ab}$ \\
\hline Setembro & $12,97 \mathrm{Ab}$ & $2,96 \mathrm{Ba}$ & $2,05 \mathrm{Ab}$ & $0,46 \mathrm{Ba}$ & $0,31 \mathrm{Aa}$ & $0,11 \mathrm{Ab}$ & $4,40 \mathrm{Ab}$ & $1,36 \mathrm{Bb}$ \\
\hline Outubro & $15,98 \mathrm{Aa}$ & $5,87 \mathrm{Ba}$ & $2,41 \mathrm{Aa}$ & $0,91 \mathrm{Ba}$ & $0,36 \mathrm{Aa}$ & 0,31 Aa & $5,57 \mathrm{Aa}$ & $3,62 \mathrm{Ba}$ \\
\hline Novembro & $11,49 \mathrm{Ab}$ & $5,35 \mathrm{Ba}$ & $1,77 \mathrm{Ab}$ & $0,87 \mathrm{Ba}$ & $0,31 \mathrm{Aa}$ & $0,35 \mathrm{Aa}$ & $3,59 \mathrm{Ab}$ & $3,09 \mathrm{Aa}$ \\
\hline \multirow[t]{2}{*}{ Dezembro } & $9,59 \mathrm{Ac}$ & $4,96 \mathrm{Ba}$ & $1,42 \mathrm{Ac}$ & $0,68 \mathrm{Ba}$ & $0,20 \mathrm{Ab}$ & $0,26 \mathrm{Aa}$ & $3,39 \mathrm{Ab}$ & $3,01 \mathrm{Aa}$ \\
\hline & & & & & \\
\hline Mês & \multicolumn{2}{|c|}{$9,96^{* * * *}$} & \multicolumn{2}{|c|}{$9,93^{* * * *}$} & \multicolumn{2}{|r|}{$6,48^{* * * *}$} & \multicolumn{2}{|r|}{$14,57^{* * * *}$} \\
\hline Componente & \multicolumn{2}{|c|}{$270,46^{* * *}$} & \multicolumn{2}{|c|}{$278,98^{\text {**** }}$} & \multicolumn{2}{|r|}{$1,44^{\mathrm{ns}}$} & \multicolumn{2}{|r|}{$20,13^{* * *}$} \\
\hline Mês * Comp. & & $4^{* * * *}$ & & $82^{* * * *}$ & \multicolumn{2}{|r|}{$2,05^{*}$} & \multicolumn{2}{|r|}{$2,68^{* *}$} \\
\hline C.V. $(\%)$ & \multicolumn{2}{|c|}{69,02} & \multicolumn{2}{|c|}{69,33} & \multicolumn{2}{|c|}{109,90} & \multicolumn{2}{|r|}{114,73} \\
\hline
\end{tabular}

R. Árvore,Viçosa-MG, v.33, n.5, p.949-956, 2009 
Do mesmo modo que para $\mathrm{P}$, a contribuição da componente miscelânea no aporte de K é também semelhante estatisticamente à componente folha (Tabela 3), com exceção dos meses de setembro e outubro, porque são meses secos de maior aporte de folhas e menores lixiviações de K. As diferenças no aporte de K provenientes das componentes folha e miscelânea ao longo do ano eram esperadas, pela correlação desse nutriente com a precipitação, mostrando que nos meses mais chuvosos o aporte de K é menor.

$\mathrm{O}$ aporte anual de nutrientes seguiu a seguinte ordem $\mathrm{Ca}>\mathrm{K}>\mathrm{Mg}>\mathrm{P}$, considerando-se tanto o aporte via folha quanto a miscelânea. A magnitude do aporte de nutrientes através da componente folha da serapilheira foi para $\mathrm{Ca}, \mathrm{K}, \mathrm{Mg}$ e $\mathrm{P}$ de 118,$0 ; 29,1 ; 18,4$; e $2,7 \mathrm{~kg} / \mathrm{ha} / \mathrm{ano}$, respectivamente (Figura 3 ). Esses resultados se encontram dentro das variações encontradas por Nunes (1980), estudando florestas de regiões tropicais, em que no componente folha da serapilheira o conteúdo de Ca apresentou variação de 14 a 240, K de 11 a 129, Mg de 8 a 44 e P de 2 a 13 kg/ha/ano.

A contribuição do componente miscelânea no aporte anual de $\mathrm{P}$ foi significativa, principalmente quando comparada com o aporte de Ca através do componente folha da serapilheira (Tabela 3), indicando que a ciclagem de nutrientes em um ecossistema florestal, para que se mantenha equilibradamente sustentável, necessita de que toda componente do ecossistema apresente sua contribuição para otimização do processo. Para que uma floresta, que cicla através de sua serapilheira 5,28 kg/ha/ano de P (Figura 3 ) e, que apresenta imobilizado, em média, só em sua biomassa foliar, construída ao longo de anos, $12,73 \mathrm{~kg} / \mathrm{ha}$ de $\mathrm{P}$, se mantenha em equilíbrio é preciso que o solo seja forte fonte de $\mathrm{P}$ ou que ocorra ciclagem direta desse nutriente da manta orgânica, principalmente em solos muito intemperizados, que apresentam elevados valores de adsorção desse nutriente (SAMPAIO et al., 1988).

\section{CONCLUSÕES}

- A deposição de serapilheira foi de 10,07 t/ha/ano, o componente folha contribuiu com $6,74 \mathrm{t} / \mathrm{ha} / \mathrm{ano}(66,9 \%)$ e a componente miscelânea, com 3,33 t/ha/ano $(33,1 \%)$.

- A deposição de serapilheira apresentou sazonalidade, sendo maior nos períodos secos.

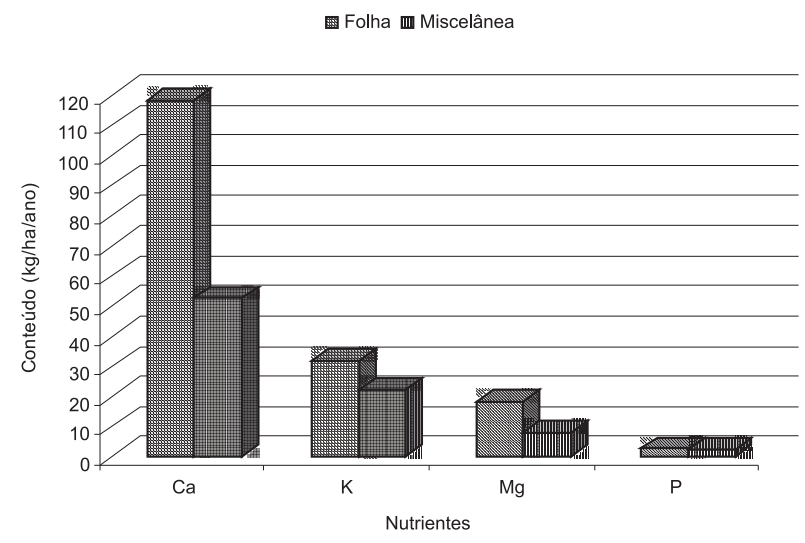

Figura 3 - Deposição anual de cálcio, magnésio, fósforo e potássio através dos componentes folha e miscelânea da serapilheira, em fragmento de Mata Atlântica, em Pernambuco.

Figure 3 - Calcium, magnesium, phosphorus and potassium input in leaf and miscellaneous in an Atlantic Forest fragment in Pernambuco, Brazil.

- O componente miscelânea da serapilheira não apresentou sazonalidade, entretanto essa sazonalidade influenciou a componente folha.

- Os teores de $\mathrm{K}$ nas componentes folha e miscelânea foram menores nos períodos chuvosos.

Os teores dos nutrientes $\mathrm{Ca}, \mathrm{Mg}, \mathrm{K}$ e $\mathrm{P}$ na serapilheira foram de 15,$73 ; 2,42 ; 4,74 ;$ e $0,50 \mathrm{~g} / \mathrm{kg}$, respectivamente, sendo o aporte de 170,$7 ; 26,4 ; 49,7$; e $5,2 \mathrm{~kg} / \mathrm{ha} / \mathrm{ano}$, respectivamente.

A contribuição do componente miscelânea da serapilheira na ciclagem de $\mathrm{P}$ foi de aproximadamente $50 \%$ do aporte total desse nutriente.

\section{AGRADECIMENTOS}

Ao Comando Militar do Nordeste, pela cessão da área onde foram instalados os equipamentos para coleta dos dados desta pesquisa.

\section{REFERÊNCIAS}

ANDRADE, A. G.; CABALLERO, S. S. U.; FARIA, $S$. M. Ciclagem de nutrientes em ecossistemas florestais. Rio de Janeiro: Embrapa, 1999. 22p.

R. Árvore, Viçosa-MG, v.33, n.5, p.949-956, 2009 
BAtAGliA, O. C. et al. Métodos de análise química de plantas. Campinas: Instituto Agronômico de Campinas, 1983. 48p.

(BoletimTécnico, 78).

BRAGA, J. M.; DEFELIPO, B. V. Determinação espectrofotométrica de fósforo em extrato de solo e material vegetal. Revista Ceres, v.21, n.1, p.73$85,1974$.

CUNHA, G. C. et al. Dinâmica nutricional em floresta estacional decidual com ênfase aos minerais provenientes da deposição da serrapilheira. Ciência Florestal, v.3, n.1, p.19-39, 1993.

DIAS, H. C. T.; OLIVEIRA FILHO, A. T. Variação temporal e espacial da produção de serapilheira em uma área de floresta estacional semidecídua Montana em Lavras-MG. Revista. Árvore, v.21, n.1, p.11-26, 1997.

\section{EMPRESA BRASILEIRA DE PESQUISA}

AGROPECUÁRIA - EMBRAPA. Centro Nacional de Pesquisas de Solos. Sistema brasileiro de classificação de solos. Brasília: 1999. 412p.

FERREIRA, R. L. C. et al. Deposição e acúmulo de matéria seca e nutrientes em serrapilheira em um bosque de sabiá (Mimosa caesalpiniifolia Bentth.). Revista Árvore, v.31, n.1, p.7-12, 2007.

FREITAS, V. L. C. Massa de serapilheira em 11 matas da faixa úmida costeira de Pernambuco. 1990. 65f. Dissertação (Mestrado em Botânica) - Universidade Federal Rural de Pernambuco, Recife, 1990.

GOlley, F. B. et al. Ciclagem de minerais em um ecossistema de floresta tropical úmida. São Paulo: EPU-EDUSP, 1978. 256p.

GONZALEZ, M. I. M.; GALLARDO, J. F. El efecto hojarasca: una revisión. Anales de Edafologia y Agrobiologia, v.41, n.5/6, p.1129-1157, 1982.
HAAG, H. P. Ciclagem de nutrientes em florestas tropicais. Campinas: Fundação Cargil, 1985. 114p.

HINKEL, R.; PANITZ, C. M. N. Estudo comparativo de serapilheira de uma área de Mata Atlântica e de um povoamento de Pinus elliotti Engelm. var. elliotti na Ilha de Santa Catarina, Brasil. Biotemas, v.1, n.12, p.67-93, 1999.

KLINGE, H. Preliminary data on nutrient release from decomposing leaf litter in a neotropical rain forest. Amazoniana, v.6, p.193-202, 1997.

MENGEL, K.; KIRKBY, E. A. Principles of plant nutrition. Bern: International Potash Institute, 1982. 655p.

NUNES, K. S. Ciclagem de nutrientes na mata de Dois Irmãos - Recife. 1980. 123f. Dissertação (Mestrado em Botânica) Universidade Federal Rural de Pernambuco, Recife, 1980.

PRITCHetT, W. L. Properties and management of forest soils. New York: J. Wiley, 1979. 500p.

SAMPAIO, E. V. S. B.; NUNES, K. S.; LEMOS, E. E. P. Ciclagem de nutrientes na mata de Dois Irmãos (Recife-PE) através da queda de material vegetal. Pesquisa Agropecuária

Brasileira, v.23, n.10, p.1055-1061, 1988.

SCHUMACHER, M. V. et al. Retorno de nutrients via deposição de serrapilheira em um povoamento de Acácia-Negra (Acacia mearnsii De Wild) no Estado do Rio Grande do Sul. Revista Árvore, v.27, n.6, p.791-798, 2003.

VELOSO, H. P.; GÓES FILHO, L. Fitogeografia brasileira, classificação fisionômica ecológica da vegetação neotropical. Salvador: Projeto RADAMBRASIL, 1982. 80p. (Boletim Técnico, 1). 